\title{
Pseudomonas makes its own bed
}

DOI:

10.1038/nrmicro1683

URLs

Pseudomonas aeruginosa http://www.ncbi.nlm.nih.gov/ entrez/query.fcgi?db=genome prj\&cmd=RetrieveEdopt=Over viewElist_uids=12339
The opportunistic and sometimes deadly pathogen Pseudomonas aeruginosa subverts the organization of epithelial cells to generate basolateral membrane patches through which it can enter cells in this all-important defensive barrier, according to research recently published in the Journal of Cell Biology.

Polarized epithelial cells, which line many internal organs, form a single layer of cells that are joined by tight junctions. The cells are highly organized, with an apical surface (AP) that faces the lumen (external) and a basolateral (BL) surface that lines tissues (internal) (see the figure). Crossing epithelia is often the first step in microbial pathogenesis and leads to tissue and organ damage through pathogen dissemination. Some pathogenic bacteria induce epithelial cells to ingest them by phagocytosis, whereas others inject effectors into host cells that rearrange the cytoskeleton and facilitate pathogen entry. Opportunists such as $P$. aeruginosa, however, can only breach damaged epithelia. The latest research from Joanne Engel's laboratory has used a model system of polarized Madin-Darby canine kidney cells to probe just how this pathogen enters epithelial cells.

Phosphatidylinositol 3,4,5-triphosphate (PIP3) is found exclusively at the BL surface, and previous work by the same authors revealed that inserting PIP3 into the AP surface converted it to a BL surface by activating transport of phosphatidylinositol 3-kinase (PI3K) across the cell in vesicles (transcytosis). When the function of PI3K, which generates PIP3, is disrupted, $P$. aeruginosa cannot enter epithelial cells. These data, along with the knowledge that receptor-mediated phagocytosis of other pathogens (Yersinia and Listeria spp.) is known to depend on PI3Ks, justified further scrutiny of a role for phosphoinositides in $P$. aeruginosa entry.

Here, building on previous work and using two different probes for PIP3, Kierbel et al. showed that binding of $P$. aeruginosa to the AP surface of epithelial cells redistributed PIP3 from the BL surface to the AP site that the bacterium had bound to. Moreover, scanning electron microscopy showed that protrusions of the AP surface occurred where the bacteria had attached. PI3K was also redistributed from the BL surface following bacterial binding and sequestered beneath the membrane protrusions. These bacteria-induced membrane protrusions were enriched for BL markers, including p58, $\beta$-catenin and $\beta 1$-integrin but lacked AP markers, indicating specific membrane alterations. Kierbel et al. then specifically labelled BL proteins and used pulse-chase experiments to show that BL proteins moved by transcytosis to the AP surface after bacterial binding. The receptor for $P$. aeruginosa - which is still unknown, although the cystic-fibrosis membrane receptor and asialoganglioside are candidates - could be located at bacterial attachment sites, which are usually found near tight junctions.

The authors speculate that $P$. aeruginosa subverts membrane organization most readily in cells that are already injured and therefore poorly polarized, which might be one reason why this opportunist can only get in when the doorway is already partly opened by cell injury.

Susan Jones

ORIGINAL RESEARCH PAPER Kierbel, A. et al. Pseudomonas aeruginosa exploits a PIP3dependent pathway to transform apical into basolateral membrane. J. Cell Biol. 177, 21-27 (2007)

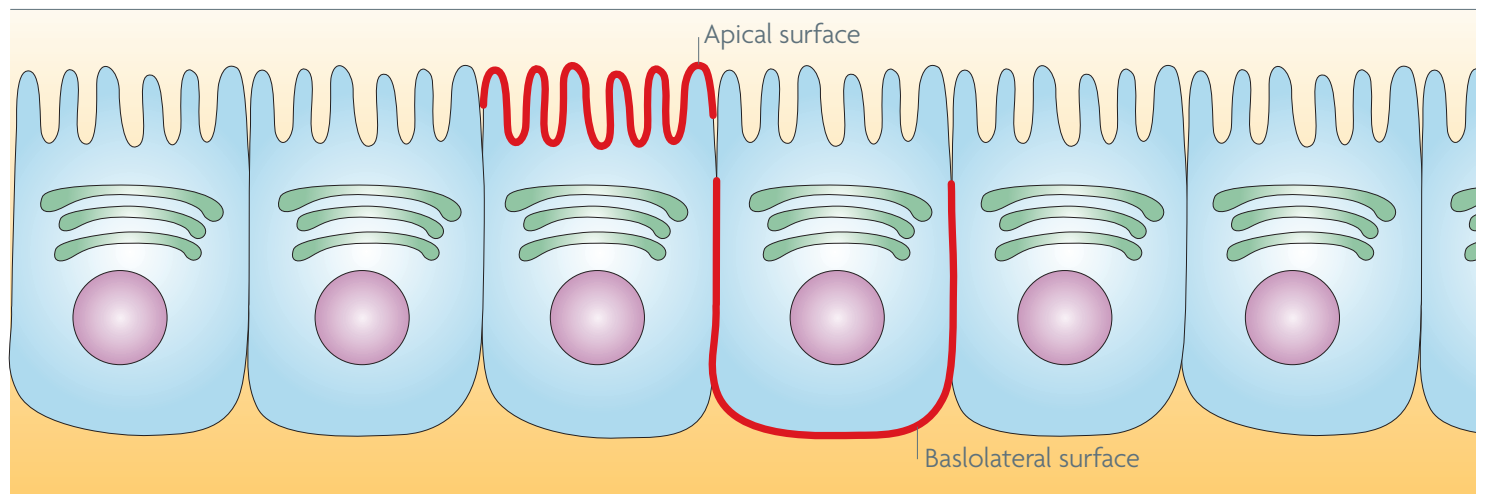

